\title{
Fermiab
}

\section{STATUS OF SEARCHES FOR HIGGS AND PHYSICS BEYOND THE STANDARD MODEL AT CDF}

\author{
D. TSYBYCHEV * \\ Department of Physics \\ University of Florida, Gainesville, FL, 32611-8440 \\ E-mail: tsybych@fnal.gov
}

FOR CDF COLLABORATION

This article presents selected experimental results on searches for Higgs and physics beyond the standard model (BSM) at the Collider Detector at Fermilab (CDF). The results are based on about $350 \mathrm{pb}^{-1}$ of proton-antiproton collision data at $\sqrt{s}=1.96 \mathrm{TeV}$, collected during Run II of the Tevatron. No evidence of signal was found and limits on the production cross section of various physics processes BSM are derived.

\section{Introduction}

A wide range of predicted phenomena in the Standard Model (SM) of particles physics has been remarkably confirmed by many experiments over the past decades. However, the central piece of the SM, Higgs boson, has not been observed so far. Furthermore, there are many fundamental questions that SM does not address. For example, the origin of the electro-weak symmetry breaking, hierarchy of scales, unification of gauge interactions and the nature of gravity are left unexplained in SM. In addition, recent cosmological observations indicate that SM particles account only for $\sim 4 \%$ of the matter of the Universe.

Several extensions to the SM are proposed to resolve these issues. These extensions include supersymmetry (SUSY), grand unified theories (GUT), technicolor (TC), extra dimensions (ED), etc. The phenomenology of these extensions is very rich, with some signatures very different from SM processes. However, masses, production cross-sections and decay branching ratios of particles in such theories are unknown and highly model-dependent,

${ }^{*}$ Currently at Department of Physics, State University of New York, Stony Brook, NY, 11794-3800 
which eventually complicates the prediction of experimental signatures. Examples of such signatures from physics BSM can be large missing transverse energy $\left(\not_{\mathrm{T}}\right)$, same sign leptons, multiple photons, heavy flavor jets or some combination of several of those. Thus two different approaches can be adopted. The most general searches look for any deviations from the SM. In this approach all measurable signatures and kinematical phase space are scanned and the results can be interpreted based on some model. However those searches are not fully efficient for a particular model. An alternative approach is to search for specific signals suggested by particular model in which some theoretical assumptions are also made to reduce the number of free parameters.

At $\mathrm{CDF}^{1}$ we search for evidence of SM Higgs and physics BSM in $p \bar{p}$ collisions at $\sqrt{s}=1.96 \mathrm{TeV}$. In this paper we report on recent results for searches based on about $350 \mathrm{pb}^{-1}$ collected since the start of Run II of the Tevatron in March 2001.

\section{Searches for Higgs Boson}

The Higgs boson is crucial to our understanding of the electro-weak symmetry breaking and mass generation of the gauge bosons and fermions. In addition, the mass of the Higgs boson may indicate the scale of new physics. The search for the SM Higgs boson is one of the major goals of the Tevatron Run II program.

\subsection{Searches for Standard Model Higgs Boson}

At Tevatron the dominant Higgs boson production mechanism ${ }^{2}$ is through gluon-gluon fusion. For light Higgs $\left(M_{H}<135 \mathrm{GeV} / c^{2}\right)$ the dominant decay channel is to $b \bar{b}$, which results in a signature of the two heavy flavor jets. However, this final state is dominated by the large SM QCD multi-jet production. Therefore, for the light Higgs CDF concentrates on channels where it is produced in association with $W$ or $Z$ bosons. On the other hand, for Higgs masses greater than $135 \mathrm{GeV} / c^{2}$, the Higgs boson is predicted to decay predominantly to $W^{+} W^{-}$pairs, in which case $g g \rightarrow H$ process is considered.

A search for light Higgs was performed in $p \bar{p} \rightarrow W^{ \pm} H \rightarrow l^{ \pm} \nu b \bar{b}$ channel using $162 \mathrm{pb}^{-1}$, by looking for a peak in the dijet invariant mass distribution in the two jet events. Candidate events were selected by requiring an energetic isolated lepton $\left(E_{T}>20 \mathrm{GeV}\right.$ for electrons or $p_{T}>20 \mathrm{Gev} / c$ for muons) in the central detector $(|\eta|<1)$, two jets with $E_{T}>15 \mathrm{GeV}$ and 
$E_{\mathrm{T}}>20 \mathrm{GeV}$. At least on of the two jets was required to be heavy-flavor tagged. The expected number of SM background events was $60.55 \pm 4.43$, coming from $W b \bar{b}, W c \bar{c}, W c, t \bar{t}$ processes and events with light flavored jets mis-identified as $b$-jets. After applying all selection cuts, 62 events were observed. The $95 \%$ C.L. upper limit on the cross section times branching ratio as a function of the Higgs mass is shown in the Figure 1.

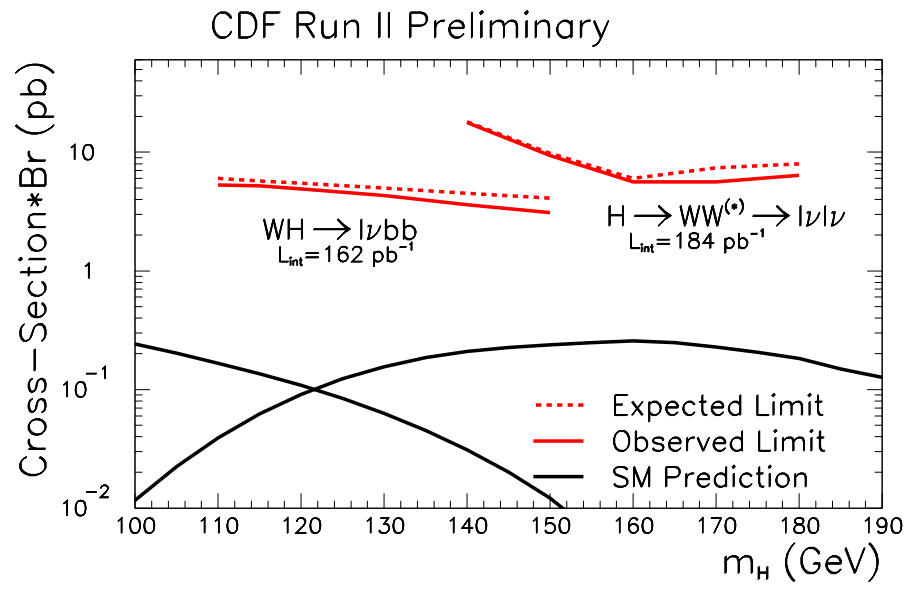

Figure 1. The $95 \%$ C.L. upper limit on the SM production cross section times branching ratio as a function of the Higgs boson mass from $W^{ \pm} H->l^{ \pm} \nu b \bar{b}$ and $H \rightarrow W W$ analyses.

A search was also performed for Higgs boson decaying to $W^{+} W^{-}$pairs in dileptons and $\not_{\mathrm{T}}$ final state with $200 \mathrm{pb}^{-1}$ of data. The candidate events were selected by requiring two oppositely charged, high- $p_{T}$, isolated leptons (electrons or muons) and large missing $\not_{\mathrm{T}}\left(\not_{\mathrm{T}}>25 \mathrm{GeV}\right)$. The $\not_{\mathrm{T}}$ was required not to be parallel to either of leptons, in order to ensure that it was well measured. Events consistent with the $Z$ boson mass were removed. In addition, we vetoed events with reconstructed jets above $E_{T}>15 \mathrm{GeV}$, to suppress diboson production background. Finally, to discriminate possible Higgs signal from $W W$ background we exploit the fact the Higgs boson is fundamental spin-zero particle, which results in smaller invariant mass of 
the two leptons $m_{l l}$. Therefore, events with small $m_{l l}$ were selected and azimuthal angular separation between the leptons was fitted using maximum likelihood method, to extract 95\% C.L. limit on production cross section times branching ratio.

Figure 1 summarizes our current sensitivity for SM Higgs in both $H \rightarrow$ $W^{+} W^{-}$and $W H$ channels. Currently, no limit on the Higgs mass can be set as these searches are statistics limited.

\subsection{Search for MSSM Neutral Higgs Boson at large tanß}

The Minimum Supersymmetric extension to the Standard Model predicts the existence of five Higgs particles, three neutral and two charged. The coupling of the neutral pseudo-scalar Higgs boson $(A)$ to the third generation fermion can be enhanced by a factor of $\tan \beta$ (the ratio of the vacuum expectation values of the two Higgs fields and it is a free parameter in the theory) relative to the SM. Thus, the production cross section for $A$ will scale as $\tan ^{2} \beta$. While the branching ratio of $A \rightarrow b \bar{b}$ dominates ( $\left.90 \%\right)$, a search in this channel is very difficult due to overhelming multi-jet background. Therefore, if $A$ is light, the $A \rightarrow \tau \tau$ decay mode ( $8 \%$ ) becomes interesting. This mode is an important part of the MSSM Higgs search at CDF.

CDF has searched for the $A \rightarrow \tau \tau$ at high $\tan \beta$ in about $200 p b^{-1}$ of data, collected with a set of dedicated $\tau$-triggers. The signal consists of a tau pair, where one of the taus decays leptonicaly, while the other decays hadronicaly. After initial selection of the events with two reconstructed tau candidates, the multi-jet background was suppressed by requiring large $H_{T}$, which is defined as a scalar sum of momenta of tau-decay products and $\not_{\mathrm{T}}$ . In addition, the $B_{\mathrm{T}}$ was required not to point in direction opposite to the tau decay products. After all of the selection cuts were applied, 236 events were observed, and the expected number of the SM background events was $263.6 \pm 30.1$. The dominant source of the background was from $Z \rightarrow \tau \tau$. To further discriminate signal from background and set a limit on production cross section a mass-like variable $m_{v i s}$ was constructed using the four momentum of the lepton, the four-momentum of visible decay products of hadronicaly decaying tau and $\boldsymbol{\#}_{\mathrm{T}}$. Figure 2 shows $m_{v i s}$ for remaining events. Maximum binned-likelihood fit was performed to the $m_{v i s}$ to extract Higgs signal. Observed limit on the production cross section times branching ratio at 95\% C.L. for the neutral pseudo-scalar Higgs is shown in Figure 3 along with expected limit from pseudo-experiments. Observed 
limit is about order of magnitude higher than theoretically predicted cross section. The sensitivity of this analysis is expected to improve with higher accumulated statistics.

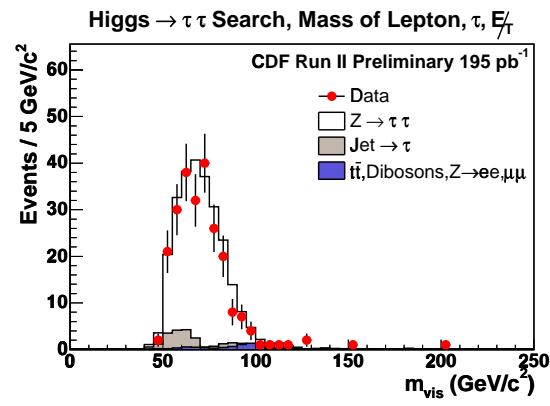

Figure 2. Observed partially reconstructed ditau mass and contribution from various SM background processes.

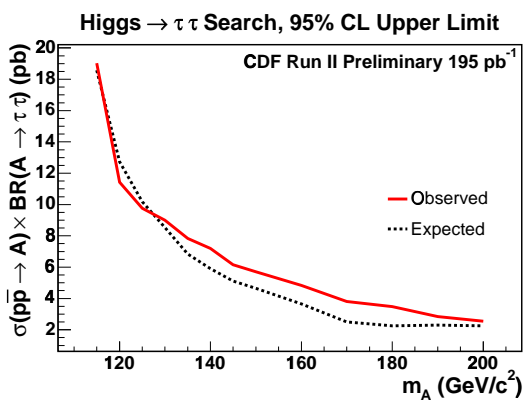

Figure 3. Observed and expected upper limit at $95 \%$ C.L. on the $\sigma(p \bar{p} \rightarrow$ $A) \times \operatorname{BR}(A \rightarrow \tau \tau)$.

\subsection{Searches for Doubly Charged Higgs Boson}

The existence of the doubly charged Higgs boson $H^{ \pm \pm}$is predicted in several left-right (L-R) symmetry ${ }^{3}$ breaking models with Higgs triplets. In some L-R SUSY ${ }^{4}$ models Higgs boson can be produced in a mass range accessible at Tevatron $\left(\sim 100 \mathrm{GeV} / c^{2}-1 \mathrm{TeV} / c^{2}\right)$. The $H^{ \pm \pm}$may be produced at the Tevatron in pairs ${ }^{5}$ via $Z / \gamma^{*}$ exchange, or singly via $W W$ fusion or $W$ Drell-Yan. The experimental signature of the $H^{ \pm \pm}$will be a pair of same sign leptons ${ }^{6}$, if the Yukawa couplings $\left(h_{l l^{\prime}}\right)$ of $H^{ \pm \pm}$to charged leptons is in the range $10^{-5}<h_{l l^{\prime}}<0.5$ (which corresponds to narrow resonances that decay promptly). Otherwise, $H^{ \pm \pm}$appears as a stable particle and decays outside the detector volume.

The search for a pair of long-lived $H^{ \pm \pm}$was done with $206 \mathrm{pb}^{-1}$ of data and concentrated on selecting events with isolated muon and an additional high momentum track with $p_{T}>20 \mathrm{GeV} / c$. The fact that energy loss due to ionization is proportional to the square of the particle charge is further exploited, and both muon and track in this analysis were required to be highly ionizing in the drift chamber. Zero events were observed, which was consistent with expectation from SM background processes. The experimental limit on the $H^{ \pm \pm}$production cross section at 95\% C.L along with theoretical cross section are shown in Figure 4. 
The search for the prompt $H^{ \pm \pm} 7$ was performed in $H^{ \pm \pm} \rightarrow e^{ \pm} e^{ \pm}$, $H^{ \pm \pm} \rightarrow \mu^{ \pm} \mu^{ \pm}$and $H^{ \pm \pm} \rightarrow e^{ \pm} \mu^{ \pm}$channels, using a data sample of $240 \mathrm{pb}^{-1}$. This search was performed in the mass window of $\pm 10 \%$ around a given $H^{ \pm \pm}$mass ( $\sim 3 \sigma$ of the detector resolution). No events were observed after applying the selection cuts in the mass region $m_{H^{ \pm \pm}}>80 \mathrm{GeV} / c^{2}$ $\left(m_{H^{ \pm \pm}}>100 \mathrm{GeV} / c^{2}\right.$ for electron pair channel), which is consistent with the SM background expectation. Figure 5 shows the $H^{ \pm \pm}$mass limits for different $h_{l l^{\prime}}$ couplings in the mass-coupling plane.

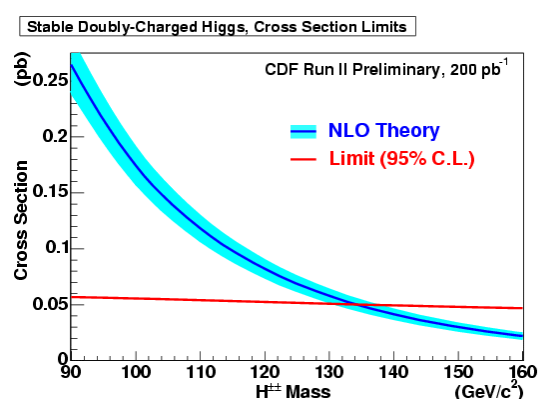

Figure 4. Experimental limit on the $H^{ \pm \pm}$production cross section at $95 \%$ C.L compared with the thoretical expctations.

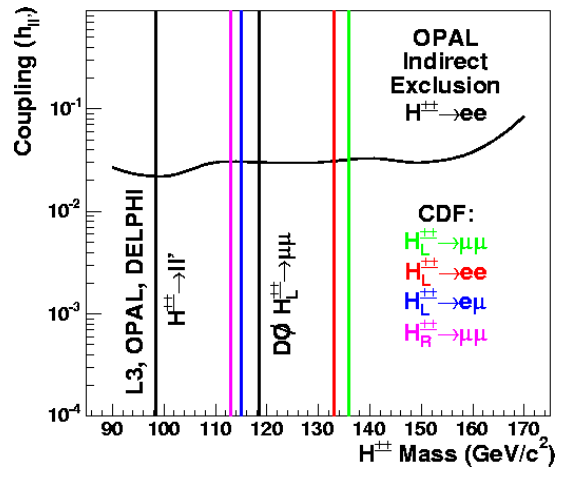

Figure 5. The $H^{ \pm \pm}$lower mass limits versus lepton couplings $\left(h_{l l^{\prime}}\right)$ from prompt $H^{ \pm \pm}$analysis, assuming exclusive decay to a given dilepton pair.

\section{Search for Scalar Bottom Quarks from Gluino Decays}

Supersymmetry solves the fine tuning problem associated with the SM Higgs mass and provides a framework for unification of the fundamental interactions. SUSY is a larger space-time symmetry that relates bosons and fermions, so that every SM particle has a supersymmetric partner with spin difference $1 / 2$. In addition a new conserved quantum number is introduced to avoid Lepton $(L)$ and Baryon $(B)$ number violation. $R$-parity is a multiplicative quantum number, defined as $R=(-1)^{3(B-L)+2 S}$ where $S$ is the spin of the particle. For the SM particles $R=1$, whereas $R=-1$ for the SUSY partners. If $R$-parity is conserved SUSY particles would be produced in pairs in collider experiments, and decay to stable final state particles: the Lightest Supersymmetric Particle (LSP) and SM quarks and 
leptons. The electrically neutral LSP is a candidate for cold dark matter. The SUSY partners of the left-handed and right-handed fermions, can mix to form mass eigenstates. For scenarios with large $\tan \beta$, the mixing in the third generation of supersymmetric particles can be substantial. In some SUSY models ${ }^{8}$, the lighter scalar-bottom quark $\left(\tilde{b}_{1}\right)$ mass eigenstate can be significantly lighter than other SUSY particles.

CDF performed a search for $\tilde{b}_{1}$ from gluino $(\tilde{g})$ decays in a $R$-parity conserving SUSY scenario with $m_{\tilde{g}}>m_{\tilde{b}_{1}}$ using $156 \mathrm{pb}^{-1}$ of data. In this scenario, gluinos are pair produced and they decay into $\tilde{g} \rightarrow \tilde{b}_{1} b$ with a $100 \%$ branching ratio, followed by the sequential decay of the $\tilde{b}_{1} \rightarrow b \tilde{\chi}_{1}^{0}$, where $\tilde{\chi}_{1}^{0}$ is the lightest neutralino and the LSP. Since neutralinos escape detection, this gives a signature of four $b$-jets and $E_{\mathrm{T}}$. Two analyses were performed using exclusive single $b$ tagged events and inclusive double $b$-tagged events. Final selection cuts require $\boldsymbol{H}_{\mathrm{T}}>80 \mathrm{GeV}$ and no reconstructed leptons. In both analyses, backgrounds are dominated by top quark pair production and $W / Z$ boson production in association with jets. In the single-tag analysis $16.4 \pm 3.6$ events were expected and 21 were observed in data. Whereas, in double-tag analysis $2.6 \pm 0.7$ events were expected and 4 observed. No evidence of new physics was observed and limits were set in gluino-sbottom mass plane as shown in Figure 6.

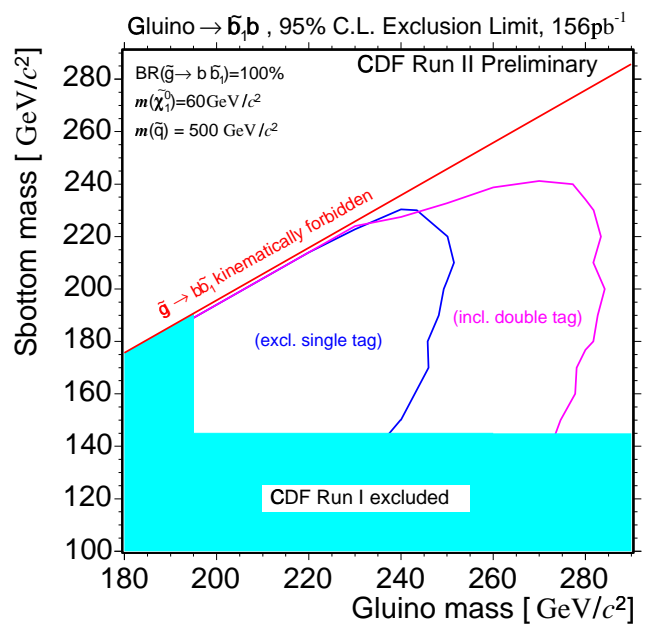

Figure 6. The 95 C.L. exclusion region in the $m_{\tilde{g}}$ and $m_{\tilde{b}_{1}}$ plane from the search for sbottom quarks from gluino decay. 


\section{Searches for Extra Dimensions}

Current string theory proposes that as many as seven new dimensions may exist and the geometry of these extra dimensions are responsible for why gravity is so weak. In recent years a number of models with extra dimensions were proposed ${ }^{9}$ to solve the hierarchy problem. In particular Randall-Sundrum (RS) model ${ }^{10}$ proposes a non-factorisable geometry in $5-D$ space, with a constant negative curvature. The extra dimension is warped by an exponential factor, which determines the masses and couplings of Kaluza-Klein (KK) states. The spectrum of the KK states is discrete and they appear as narrow resonances. The properties of the RS model are determined by the ratio of $k / M_{P l}$, where $k$ is a curvature scale of the theory and $M_{P l}$ is the Plank mass.

The CDF has searched for a RS graviton signal of the first excited KK state in diphoton channel, using $345 \mathrm{pb}^{-1}$ data sample. The analysis required two photons with $p_{T}>15 \mathrm{GeV} / c$ in the central region of the detector and with invariant mass greater than $30 \mathrm{GeV} / c^{2}$. The dominant SM backgrounds to this search are the diphoton production and milti-jet production, where jets fragment into $\pi^{0}$ and pass photon selection cuts. The diphoton mass spectrum observed in data agrees well with SM prediction and is shown in Figure 7. The limits on a RS graviton particle of the first excited state was placed using a $3 \sigma$ mass window around a given graviton mass point in a range between $200 \mathrm{GeV} / c^{2}$ and $900 \mathrm{GeV} / c^{2}$. Figure 8 shows the excluded region in the RS graviton mass and warp factor plane. Also shown the CDF results for the RS graviton searches from the dimuon and dielectron channels.

\section{Searches for Leptoquarks}

The remarkable symmetry between quarks an leptons in the SM suggests that some more fundamental theory may exist, which allows interactions between them. Such interactions are mediated by a new type of particle, a leptoquark (LQ). Leptoquarks are hypothetical color-triplet bosons carrying both lepton and baryon quantum numbers that are predicted in many extensions of the standard model (e.g. grand unification models, technicolor, and supersymmetry with $R$-parity violation) ${ }^{11}$. The Yukawa coupling of the leptoquark to a lepton and quark and the branching ratio to a charged lepton, denoted by $\beta$, are model dependent. Usually it is assumed that leptoquarks couple to only one generation to accommodate experimental constraints on flavor-changing neutral currents, which allows 


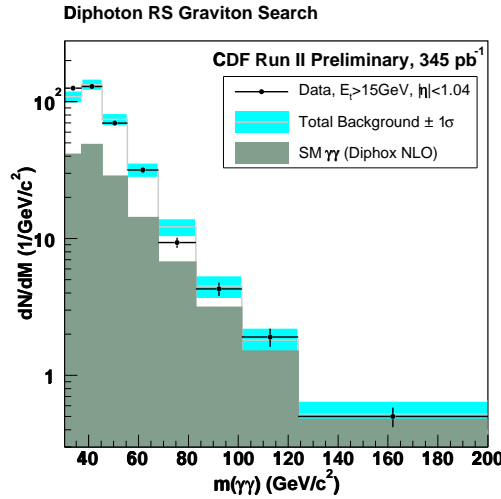

Figure 7. The observed diphoton mass spectrum compared with the SM prediction.

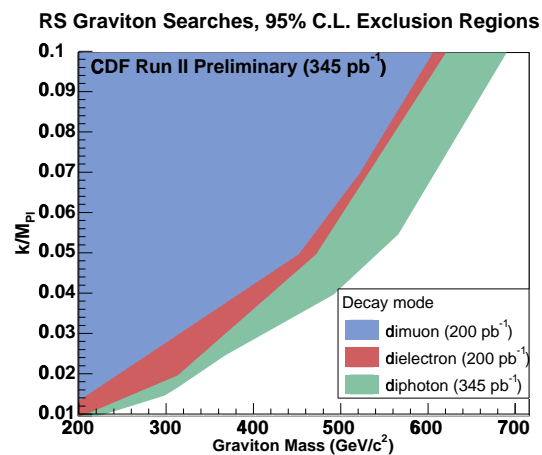

Figure 8. The limits on the RandallSundrum graviton model displayed as a function of graviton mass and warp factor for searches in the diphoton and dileptons channels.

one to classify leptoquarks as first-, second-, or third-generation. In $p \bar{p}$ collisions, leptoquarks can be produced in pairs via the strong interaction through $g g$ fusion or $q \bar{q}$ annihilation.

Several analyses were carried out at CDF to search for first (second) generation leptoquarks: in $L Q \overline{L Q} \rightarrow e e(\mu \mu) q \bar{q}(\beta=1)$ and in $L Q \overline{L Q} \rightarrow e(\mu) \nu q \bar{q}(\beta=0.5)$ channels in $200 \mathrm{pb}^{-1}$ data sample. In addition the search was performed in $L Q \overline{L Q} \rightarrow \nu \bar{\nu} q \bar{q}(\beta=0)$ channel, which is sensitive to the leptoquarks of all three generations. The main sources of the SM backgrounds in these searches are represented by production of a vector boson $(Z$ or $W$ ) accompanied by jets and top quark production. No evidence for the existence of leptoquarks was observed. Upper limit on leptoquark pair-production cross sections were placed at 95\% C.L. and lower limits on their masses were derived. The results are summarized in Table 1.

\section{Summary}

We reviewed some of the most recent results from the CDF on the searches for SM Higgs and other new phenomena at the Tevatron. No evidence for new physics has been observed so far and limits on different models were derived. The new results already have surpassed many Run 1 results. The sensitivity will improve as CDF detector continues to take data and many 
new results should be expected soon in the data set of about $0.5 \mathrm{fb}^{-1}$. The Tevatron remains the energy frontier of collider searches for new physics and provides the best opportunity for finding evidence for physics BSM before LHC starts.

\section{References}

1. CDF Collaboration, FERMILAB-PUB-96/390-E.

2. A. Stange, W. Marciano and S. Willenbrock, Phys. Rev. D49, 1354 (1994).

3. R. N. Mohapatra and J. C. Pati, Phys. Rev. D11, 566 (1975).

4. C. S. Aulakh, A. Melfo and G. Senjanovic, Phys. Rev. D57, 4174 (1998).

5. R. N. Mohapatra and G. Senjanovic, Phys. Rev. Lett. 44, 912 (1980).

6. J. F. Gunion et al. Phys. Rev. D40, 1546 (1989).

7. D. Acosta et al. [CDF Collaboration], arXiv:hep-ex/0406073; Submitted to Phys. Rev. Lett.

8. A. Bartl, W. Majerotto and W. Porod, Z. Phys. C64, 499 (1994) [Erratum C68, 518 (1995)].

9. For a review, see J. Hewett and M. Spiropulu, Annu. Rev. Nucl. Part. Sci. 52, 397 (2002).

10. L. Randall, R. Sundrum, Phys. Rev. Lett. 833370 (1999).

11. W. Buchmüller, R. Rückl and D. Wyler, Phys. Lett. B191, 442 (1987) [Erratum B448, 320 (1999)]. 
Table 1. Lower limits on the first- and second-generation leptoquark mass at $95 \%$ C.L. from CDF Run II.

\begin{tabular}{ccc}
\hline Generation & $\beta$ & $\begin{array}{c}95 \% \text { C.L. lower mass limit } \\
\left(\mathrm{GeV} / c^{2}\right)\end{array}$ \\
\hline \multirow{2}{*}{1} & 1 & 230 \\
& 0.5 & 176 \\
& 0 & 117 \\
\hline \multirow{2}{*}{2} & 1 & 242 \\
\hline
\end{tabular}

